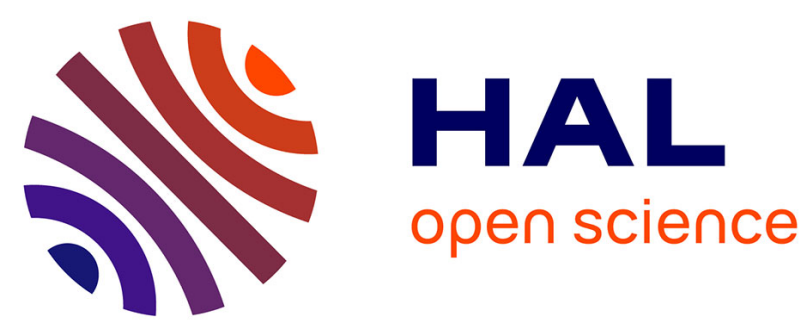

\title{
Narcissisme, créativité et prédation dans les groupes institués
}

\author{
Georges Gaillard, Guy Gimenez
}

\section{To cite this version:}

Georges Gaillard, Guy Gimenez. Narcissisme, créativité et prédation dans les groupes institués. Bulletin de psychologie, 2014, Psychologie clinique des groupes et des médiations thérapeutiques, de l'institution et du lien social, 66 (5256, fascicule 4), pp.323-332. 10.3917/bupsy.526.0323 . hal01340264

\section{HAL Id: hal-01340264 https://hal-amu.archives-ouvertes.fr/hal-01340264}

Submitted on 6 Sep 2016

HAL is a multi-disciplinary open access archive for the deposit and dissemination of scientific research documents, whether they are published or not. The documents may come from teaching and research institutions in France or abroad, or from public or private research centers.

$$
\text { Copyright }
$$

L'archive ouverte pluridisciplinaire HAL, est destinée au dépôt et à la diffusion de documents scientifiques de niveau recherche, publiés ou non, émanant des établissements d'enseignement et de recherche français ou étrangers, des laboratoires publics ou privés. 


\title{
Narcissisme, créativité et prédation dans les groupes institués
}

\author{
GAILLARD Georges* GIMENEZ Guy**
}

\begin{abstract}
« L'amour-propre est un ballon gonflé de vent dont il sort des tempêtes quand on y fait une piqûre. » (Voltaire, 1748).
\end{abstract}

\begin{abstract}
«Sans qu'elle ait à le poser comme but, la démarche analytique va rencontrer les figures intimes de la barbarie : l'appétit cannibalique, le désir de meurtre, l'inceste, les appétences sadiques et masochistes, l'ambivalence inscrite au creux des sentiments dits les plus nobles; et encore, les identifications au père de la horde, à la mère omnipotente. Au dedans et au dehors circulent les figures d'emprise et des mouvements pulsionnels de même nature. Et ce sont ces positions subjectives singulières et communes, individuelles et politiques, que le travail psychanalytique sort de leur activité clandestine. »(Zaltzman, 2003, p. 237).
\end{abstract}

Dans le champ de l'intervention institutionnelle, dans les secteurs du soin et du travail social (régulations d'équipe, analyse de la pratique, intervention de formation...), la violence des phénomènes groupaux rencontrés a conduit nombre de praticiens du champ de la psychologie à se doter d'une grille de lecture, à même de donner quelque lisibilité et d'ordonner la complexité en jeu. La clinique psychanalytique des groupes institués offre un tel étayage et permet de se dégager de la puissance des affects et des transferts mobilisés à l'occasion de ces interventions en institution (qu'il s'agisse d'interventions réalisées à partir d'une position interne ou externe aux établissements). Rappelons que la violence, inhérente à ces institutions du soin et du travail social, résulte de l'appareillage entre les équipes et leurs publics spécifiques. Les " usagers " actualisent leurs symptômes (troubles psychiques, somatiques ou du registre de l'agir) sur ces scènes institutionnelles; et cette négativité, propre aux symptômes que ces institutions se sont données pour tâche d'accueillir et de transformer, s'amalgame avec la négativité inhérente au sujet, à la groupalité et aux configurations institutionnelles.

Dans l'émergence du champ de la clinique des « groupes institués " ${ }^{1}$ et des institutions, l'ouvrage L'institution et les institutions, dirigé par René Kaës et paru en 1987, a joué comme un repère légitimant. L'article, désormais classique, d'Eugène Enriquez, paru dans ce même ouvrage, a conduit à penser l'indispensable prise en compte du « travail de la mort dans les institutions ». Or, même si, par essence, une telle dimension n'en finit pas d'échapper et de devoir être refondée, une attention portée aux transformations actuelles de ces institutions nous confronte à un déni massif de ce registre de la psyché, et de la présence de

1. Par "groupe institué », nous désignons les équipes de travail, soit l'ensemble des professionnels qui sont identifiés à partir des fonctions qu'ils occupent au sein d'une institution, et de la tâche primaire pour laquelle ils sont mandatés et qu'ils ont en partage. Ce registre de la groupalité conduit à penser les dynamiques en jeu dans une perspective différente et complémentaire de ce que le travail auprès des groupes auto-référés a permis de mettre à jour (dont le Cercle d'études françaises pour la formation et la recherche : approche psychanalytique du groupe, $\mathrm{du}$ psychodrame, de l'institution [CEFFRAP], en France, a été le creuset, à partir des travaux de Didier Anzieu et de René Kaës). Les groupes institués ont ceci de particulier qu'ils doivent composer, d'une part, avec les « usagers ", auxquels leur institution a choisi de s'adresser et, d'autre part, avec une histoire, et à construire des rapports socio-politiques exigés par le vivre ensemble.

* Centre de recherche en psychopathologie et psychologie clinique (CRPPC), Université Lumière Lyon 2 (EA653).

** Centre de recherche en psychologie de la connaissance, du langage et de l'émotion (PsyCLE), Université de Provence (EA3273).

Correspondance : Georges Gaillard, Institut de psychologie, Université Lumière Lyon 2, 5 avenue Pierre Mendès France, 69500 Bron. <georges.gaillard@orange.fr> 
Thanatos. L'hypermodernité ${ }^{2}$ promeut une culture managériale, culture des résultats et des « stratégies gagnantes », qui ne s'embarrasse pas d'une négativité par trop encombrante, ni des incidences de ses actions à moyen et long terme, et qui fait fi de la complexité. La compréhension qui découlait d'un regard établi sous le primat de l'inconscient, se trouve à présent discréditée ; elle devient l'enjeu d'une volonté de rupture des filiations antérieures, précipitant une véritable crise généalogique (Gaillard, 2002).

Il convient de souligner que le fond de destructivité et de barbarie, inhérent à la constitution du sujet (Kant, 1792 ; Freud, 1933 ; Arendt, 1951 ; Zaltzman, 1998), n'est jamais totalement transformé, malgré ce que nous aimons nous donner à croire. Nous savons qu'en grande partie il est mis au silence dans les cadres (Bleger, 1966), et noué dans les liens où s'étaye le sujet. Il est notamment noué là où le sujet joue ses identifications : la scène de ses liens amoureux, et la scène où il développe sa créativité sociale, dont centralement (mais non exclusivement) la scène professionnelle. L'une de ses expressions privilégiées est constituée par le versant mortifère du narcissisme, ce qu'A. Green (1983) désigne comme narcissisme de mort, et qui fait écho à ce que Voltaire (1748) désignait par la notion « d'amour-propre » (citation posée en épigraphe). La scène institutionnelle constitue l'un des terrains d'expression privilégié du narcissique, par les effets d'image, le pouvoir qu'elle potentialise, et la complexité des configurations en jeu. Cette scène permet d'incessants masquages des positions narcissiques et de la mise en place de faux-semblant, au travers de l'impossibilité de l'assignation des propos tenus par les différents acteurs ; tout professionnel pouvant revendiquer que son propos vise une autre adresse, ou un autre niveau d'adresse que celui qui est en jeu et qui joue de la prédation et de la disqualification. Au sein de ces espaces, la parole est aisément transformée en discours, destituant alors les places de chacun et le lien de confiance qui les fondent ${ }^{3}$.

1 Selon l'expression de J.-F. Lyotard (1979), reprise par un certain nombre d'auteurs, dont J.-P. Pinel (2008).

2 «La parole s'oppose au discours, en ce qu'elle est un dire adressé, habité par l'affect. Elle témoigne d'un vécu subjectif, qui qualifie le sujet et l'autre de la relation, dans leur singularité. Le "je" se constitue dans l'adresse à un "tu". La parole suppose la qualification d'un ou de plusieurs autres sujets dans un lien de confiance. $A$ contrario, le discours est une parole vidée de l'affect et de la singularité de l'alliance où elle prend forme. Il peut alors être utilisé comme une arme de conquête (de place, de territoire) au service d'une rivalité mortifère, déniant la subjectivité de l'autre, et l'altération propre à toute rencontre authentique » (Gaillard 2012). mouvements de prédation sont la modalité la plus banale, sont fonction du métacadre social et des modalités de relations qu'il légitime. Pour éclairer cette affirmation, il suffit de penser au cadre de la guerre et à sa nécessaire légitimation par un État. Le crime est, alors, transformé en devoir. Sur ce registre de la violence légitimée des hommes et de leur plongée dans la barbarie, soulignons qu'en France, la génération, dans laquelle ont pris place les sujets, qui sont nés à partir de l'année 1945, est la première où les hommes n'ont pas eu à se livrer directement à ces atrocités-là. Ce rappel permet de prendre la mesure du fait que la conflictualité psychique et les renoncements pulsionnels, consentis par les sujets, sont bien fonction de ce qu'un régime politique potentialise comme modalité du vivre ensemble.

L'actualité de la clinique des institutions et des groupes institués, se doit de continuer de penser la négativité propre à ces configurations, les mutations sociétales en cours, et leurs implications.

Dans un premier temps, nous allons nous pencher sur la constitution des équipes de professionnels (du champ du soin et du travail social), en soulignant l'indispensable partage d'un sentiment de créativité, en tant qu'il est seul à même de permettre de nouer la négativité de chacun des professionnels ${ }^{4}$. Nous tenterons de dégager quelques conditions de l'être en groupe dans ces institutions, soit ce que les liens d'équipes supposent de conflictualité vivifiante, en ce qu'elle participe, également, à faire barrage à la destructivité de chacun. Nous reviendrons, dans un deuxième temps, sur quelques aspects de la transformation $\mathrm{du}$ contexte social actuel, en soulignant les ruptures de filiations en cours, la levée d'une limitation de la jouissance narcissique, et les incidences de ces changements sociaux sur les sujets et les équipes. Nous serons attentifs aux mouvements de prédation, d'appétit cannibalique ${ }^{5}$ en donnant quelques illustrations paradigmatiques de ces agir, dans lesquels la disqualification est au premier plan.

1 Sous ce terme de « négativité de chacun ", nous désignons ce qui relève de la pulsion de mort (dans ses aspects mortifères, de destructivité et de désubjectivation, Freud, 1920). Tout sujet, dans la construction, est appelé au travail du renoncement, soit à troquer son omnipotence imaginaire, contre un investissement par le groupe d'accueil, base du « contrat narcissique ». De manière analogue, les groupes professionnels contraignent le nouveau venu à faire groupe avec eux, en limitant son omnipotence, son désir d'emprise, sa visée de jouissance, etc. Il s'agit donc de « nouer » ce registre de la subjectivité de chacun, et de le canaliser au service de la réalisation de la tâche primaire.

$2 \quad$ En écho à la citation de Nathalie Zaltzman, posée en épigraphe. 
Précisons que notre réflexion prend appui sur une pratique d'intervention en établissement de soin et de travail social de plus de vingt ans, au titre de régulations institutionnelles, d'analyse de la pratique ou sous le couvert d'actions de formation à l'adresse d'équipes instituées. C'est de se retrouver aux prises avec des modalités transférentielles, dans lesquelles les sentiments de confusion le disputent aux éprouvés de sidération, que les intervenants en sont venus à privilégier une lecture qui prenne en compte les interactions entre les transformations du métacadre social, les dynamiques locales et singulières, propres à une équipe et à un établissement, et la place qui revient au sujet dans son lien à l'institution ; soit aux nouages entre les registres « intra-psychique », « inter », et « trans » subjectif.

\section{LES LIENS D'ÉQUIPE, UNE GROUPALITÉ OBLIGÉE}

Les groupes institués, les équipes de travail, se présentent (encore) comme un des lieux où le sujet « ne peut pas ne pas » composer avec d'autres dans la réalisation de la tâche primaire. Elles constituent une groupalité contrainte, une groupalité obligée. Les groupes institués tendent ainsi à devenir des lieux qui contribuent au maillage du corps social, lieux de résistance potentielle, face au primat de l'individu et de sa réalisation narcissique (Gaillard, Pinel, Diet, 2009), pour autant qu'ils parviennent à se construire comme des espaces, pour une possible créativité groupale (Barthélémy, Gimenez, Pedinelli, 2007).

Toute équipe revendique, en effet, son identité, à partir de ce qu'elle considère comme sa part de transformation, de créativité agie, sa participation au « travail de l'humain », qui n'est autre que celui de la « civilisation de la pulsion », de la Kulturarbeit ${ }^{6}$ (Freud, 1932 ; Zaltzman, 1998). C'est au travers des dispositifs de prise en charge, et de la mise en discours qui les supportent, que le narcissisme groupal émerge et se ressource. La créativité peut dès lors s'entendre comme participation à l'existence, comme manière de « poser sa marque ", et de faire œuvre - au bénéfice d'autrui, au bénéfice des différents usagers concernés par les soins ou l'accompagnement et, de façon conjointe, au bénéfice du groupe professionnel lui-même. En cela, elle rassure chacun sur le fait que le fond de destructivité qui caractérise tout sujet n'a, pour un temps, toujours pas eu gain de cause.

Pour penser un tant soit peu la complexité, à l'œuvre dans ces configurations institutionnelles, qu'avec Alain-Noël Henri $(2004,2009)$ nous

6. « Travail de la culture ».

Désignons comme les institutions de la mésinscription $^{7}$ (soin, travail social, etc.), il faut rappeler qu'elles se placent sous le primat de
Thanatos et, à partir de là, explorer les « bricolages » qui permettent aux professionnels d'être à même de faire pièce à ce travail de la mort (entre déliaison mortifère et collages confusionnants).

Le narcissisme de vie (Green, 1983) est, en effet, aussi indispensable au groupe qu'il l'est pour tout sujet. Pour que ce narcissisme se fasse « suffisamment » tempéré (dans la polysémie de la tempérance, cette ancienne dénomination de l'abstinence, et du « tempérament » musical), il n'est d'autres voies que de l'arrimer à la tâche primaire. Un tel mouvement suppose que le groupe des professionnels se prête à la déliaison mortifère, qu'il se prête aux transferts. Lorsque le groupe accède au plaisir d'œuvrer et de penser ensemble, qu'il partage les éprouvés sans s'y confondre, il est alors à même de tolérer une place pour du manque, une place pour du creux (Pontalis, $2010^{8}$ ), une place pour un féminin de liaison (Gaillard, 2008). Pour les « psychistes » qui interviennent (en interne ou en externe auprès des équipes), le travail consiste à se mettre au service de la créativité groupale, soit de veiller à fluidifier ce qui entrave ce mouvement de créativité inhérent à l'humain.

Dans toute équipe, il importe que chacun des professionnels s'identifie au groupe et se reconnaisse (a minima) dans ses appartenances, qu'il s'affile, via « le projet institutionnel », et l'organisation à laquelle ce projet a donné forme ${ }^{9}$,de manière à ce que la revendication narcissique potentiellement mortifère de chacun s'arrime au narcissisme groupal, et s'articule à la tâche primaire. C'est un narcissisme groupal de bon aloi,

1 Créé par Alain-Noël Henri (2004, 2009), ce concept permet de rendre compte du travail d'unification qu'accomplit, de façon ininterrompue, tout groupe social. Il s'agit de cet inlassable travail de restauration d'un ordre symbolique en perpétuel devenir. Les pratiques de la mésinscription désignent l'ensemble des pratiques, qui concourent à ce travail de remaillage (le soin, le travail social, l'accompagnement, etc.). Les institutions de la mésinscription désignent les structures qui servent de cadre à ce travail.

2 «Capacité d'aimer (Freud), capacité de rêver (Winnicott), capacité dépressive (Fédida), dans tout cela je vois une même origine : le creux (je préfère ce mot à celui de « manque », devenu l'objet d'un véritable culte). C'est seulement quand on consent à s'approcher de ce creux, de ce silence, puis à s'enfoncer en lui, au risque de frôler l'abîme, mais avec l'espoir d'y trouver une source

souterraine, que toutes ces capacités ont une chance de se réaliser. » (Pontalis, 2010, p. 56).

3 La mise en mots du projet qualifie (énonce la particularité, souligne l'originalité). Il induit une représentation inaugurale qui participe de l'arrimage du narcissisme à la réalisation de la tâche primaire 
qui tempère l'inflation narcissique individuelle (Gaillard et coll., 2009).

La limitation narcissique ne se constitue qu'en passant par l'investissement d'un autre. Elle se déploie en un mouvement où un sujet se rend sensible à l'être d'un autre sujet et/ou à son mal-être. À partir du moment où il se sent « concerné » (selon la formule de Lévinas, 1982), et où il est à même de s'identifier, il se met en place d'assumer une responsabilité auprès de cet autre, de " prendre en charge », de soigner, d'accompagner, etc.

Le danger qui pèse sur les groupes professionnels réside dans ces mouvements où (sous de multiples aléas : évènementiels, organisationnels, etc.) les professionnels s'éprouvent dans une menace identificatoire et identitaire ${ }^{10}$. Le détour par « l'usager » n'est plus alors perçu comme source de gratification suffisante, et l'on voit émerger le risque d'un enfermement dans un " pacte narcissique » (Kaës, 2003) auto-érotique, qui exclut ce qui vient menacer la visée de complétude narcissique, « l'obsession de la plénitude » (selon les termes d'E. Enriquez, 1999) ou, plus trivialement, la cohésion groupale.

C'est, en effet, l'hétérogénéité des visées de satisfaction libidinale des sujets et de celle d'un groupe, qui met en place un potentiel de conflictualité à même de préserver le groupe professionnel d'un tel enfermement. La confrontation des narcissismes individuels (suffisamment pacifiés) fait, alors, barrage aux menaces de " pactes narcissiques " groupaux (Gaillard et coll., 2009).

De telles mises en tension demeurent, toutefois, éminemment précaires, tant il est vrai que la conflictualité est soumise au risque permanent de dégénérer en conflit, lorsque se radicalisent les différences et que l'idéologie et l'exclusion prévalent. Le groupe peut, à tout moment, fuir l'effort que suppose le maintien de la conflictualité, en développant des mouvements d'illusion fusionnelle ; mouvements qui, immanquablement, se retournent en violences d'exclusion (en violence sacrificielles, Girard, 1982). Les remaniements nécessaires au maintien d'une structure vivante supposent de laisser une part à la déliaison créative, à la « pulsion anarchiste » (Zaltzman, 1998). C'est, dès lors, la présence d'une conflictualité suffisamment ritualisée qui potentialise les mouvements d'ouverture.

10. « Le sujet est impliqué dans un ensemble de fidélités, qui mises à mal par l'autre, l'obligent à se retourner vers ses propres identifications. [...] Les phénomènes d'identifications ne relèvent de l'identité qu'en temps de crise, de vacillement des repères ou d'irruption de la barbarie et de l'affect ou de la passion, sur la scène du politique. » (Hassoun, 1994, p. 55). l'engagement du professionnel a partie liée avec des éprouvés de sécurité interne et de solidarité groupale, qui, tous deux, supposent un don de légitimité. La présence de cet ingrédient fluctue, en fonction des configurations dynamiques et temporelles, et du degré d'inclusion et de reconnaissance mutuelle, auquel le groupe est parvenu, tant au niveau des appartenances entre pairs qu'entre professionnels de professions et de niveaux hiérarchiques différents. La légitimité autorise la prise de risque. Paul Fustier (1999, p. 159) la définit, du reste, comme « avoir le droit de faire et d'être en sécurité ». Une telle position est aux antipodes du sécurité-sécuritaire et de la déstabilisation constante qui, dans les temps actuels, de procédures d'évaluations (Diet, 2003) en démarches d'accréditation, permettent de moins en moins aux équipes de s'installer dans des liens suffisamment ombragés et silencieux et suffisamment pérennes. Les (toujours) nouvelles procédures managériales détruisent, en effet, les cultures locales (les expériences accumulées) et l'histoire où elles se sont tramées : ces bricolages singuliers où se fondent la créativité des équipes.

Lorsque, de certifications en normes «ISO », les procédures institutionnelles se standardisent, on a affaire à une destruction de la diversité. Or celle-ci est essentielle à la prise en charge des sujets « mésinscrits », du fait de l'incessante transformation des formes du malaise social, et de la nécessité, pour tout sujet et pour tout groupe, de se vivre comme unique, comme non interchangeable dans le lien à l'autre. La configuration des symptômes est corrélée avec les modalités de liens sociaux (à la tonalité d'une époque et d'un contexte), à l'intérieur d'un espace sociétal donné. Ils sont une réponse à ce qui des modalités de l'" être ensemble », objet du « contrat social », ne permet pas au sujet de s'y éprouver vivant dans sa singularité et ses appartenances.

\section{La conflictualité vivifiante}




\section{TENTATIONS FAITES AU SUJET ET MODIFICATION DU MÉTA-CADRE}

Si la constitution des équipes requiert un incessant travail du lien, en vue de faire groupe autour de la tâche primaire, il faut nous rendre attentifs à quelques-uns des aspects des mutations actuelles qui participent à leur fragilisation et à leur mise en crise.

Le refus du " négatif », évoqué dans notre propos introductif, qui caractérise la période actuelle, est potentialisé par une corrélation entre l'inflation des narcissismes individuels et un affaiblissement massif, pour ne pas dire un effondrement, des légitimités traditionnelles, une transformation des méta-cadres. Une telle affirmation confine au poncif, tant sont nombreux les travaux qui concourent à ce diagnostic (Lasch, 1979 ; Elias, 1991 ; Legendre, 1996 ; Kaës, 1996 ; Castoriadis, 1996 ; Gauchet, 1998 ; Douville, 1998 ; Benasayag, 1998 ; Melman, 2002 ; Dufour, 2002 ; etc.). Dans un tel contexte, la prédation apparaît comme l'un des syndromes majeurs de la transformation des liens sociaux et d'une configuration qui tend à mettre en place une lutte de tous contre tous. Ceci passe par une mise en chauffe de la rivalité, qui se légitime d'une « libre concurrence » et de la mise en place d'un élitisme posé comme nouvelle modalité de faire groupe - il n'est que de penser à la mise en place de «pôles », dits « d'excellence », dans le secteur médical, dans l'enseignement, dans la recherche ${ }^{11}$, etc. Cette dynamique touche l'ensemble des secteurs des activités humaines et participe de ces mouvements massifs d'exclusions sociales qui, pour nombre de sujets, se muent en auto-exclusion (Furtos, 2009). Or, c'est précisément d'exclusion et d'accrocs dans le processus de subjectivation et de symbolisation, dont s'occupent les institutions de la « mésinscription ». 
Le déni du primat du « travail de la mort», dans ces structures, n'est pas sans liens avec une « banalisation ${ }^{12}$ » de la perversion. Depuis un certain temps, l'affaire est entendue : « Il n'y a pas de mal à se faire du bien ». Chaque sujet est convié à œuvrer à son développement et à son épanouissement personnel, soit, donc, à cultiver amoureusement son narcissisme, omettant de considérer qu'il est lié à l'obligation d'en passer par un autre. Or, s'il n'est pas contraint dans l'ordre de l'altérité, ce narcissisme joue alors d'une inflation phallique et se fait mortifère ${ }^{13}$.

Sous la poussée utilitariste et néolibérale, le sujet hypermoderne est de moins en moins disposé à payer le prix du renoncement pulsionnel, il tente de s'affranchir de la dette d'altérité qui le relie à ses semblables, dans le même temps où il s'efforce de s'extraire de sa dette généalogique. Face à l'inanité d'une jouissance qu'il s'épuise à satisfaire

- véritable nouveau tonneau des Danaïdes -, il

11. Ce syndrome est, bien entendu, bien plus large que le seul monde de la recherche universitaire, où ce slogan fait florès, et conditionne l'accès aux financements.

12. Parmi les auteurs, qui partagent ce diagnostic, citons : Douville (1998), Melman (2002), Diet (2003), Henri (2004), Dufour (2009). Le «banal» n'est bien entendu pas sans écho avec la célèbre formule de Hanna Arendt (1951), qui fit scandale lors de son énoncé à l'occasion du procès d'Eichmann : « la banalité du mal ». 13. P.-L. Assoun (1994) spécifie un aspect du narcissisme comme phallique et mortifère. Une telle spécification résonne avec ce qu'A. Green (1983) dénomme « narcissisme de mort ».

En court le risque d'une dérive mélancolique (Douville, 1998).

Dans le même temps, nous assistons à une paradoxale (re)qualification forcenée de la notion d'autorité. Sous l'effet d'un volontarisme affiché, on a affaire à un autoritarisme qui ne se donne pas même la peine de voiler sa prétention : une volonté effrontée de prise de pouvoir, et le libre exercice de la jouissance qui lui est corrélé. Bien entendu, une certaine manière d'incarner et d'exercer le pouvoir dans notre pays, au niveau des instances les plus élevées de l'État, n'y est pas pour rien, même si le syndrome dépasse largement les récentes frasques d'un président «bling-bling » et de sa cour. L'adage, désormais célèbre, d'une certaine marque de cosmétique, témoigne, au mieux, de ces mutations sociales et de la tentation à laquelle est soumis tout sujet : se soustraire à la castration. «Parce que je le vaux bien!»

\section{Rupture dans les filiations}

Dans le monde du soin et du travail social, l'effondrement des légitimités antérieures et la caricaturale reprise en main, que l'on voit opérer par le biais des agences et des procédures d'accréditations et autres mainmises de l'État, passent donc par une rupture avec les filiations antérieures. Ces mutations sont perceptibles, de façon caricaturale, au niveau des transformations de la constitution des directions d'établissement et des modalités d'expression de l'autorité qui s'y développe, sous l'égide du « management gestionnaire »(Gaillard, Pinel, 2011). Il fut un temps, pas si éloigné, où les hiérarchies des établissements se constituaient majoritairement à partir de promotions et de recrutements endogames au secteur (parfois même endogames à l'établissement; ce sont, au reste, de tels éléments qui ont concouru aux retournements actuels). Des professionnels $\mathrm{du}$ secteur social devenaient directeurs de structure, sans changer de secteur ; la culture des établissements se constituait ainsi, progressivement, en réservoir d'expérience. Or, depuis quelques temps déjà, la mode est aux recrutements de responsables en complète extériorité, eu égard à ces champs d'exercice. Nous voyons apparaître des directeurs issus de l'industrie, qui prennent, par exemple, des directions de pôle de soin en addictologie, des directrices ayant fait leur carrière au sein de filières administratives, qui se retrouvent à diriger des établissements de travail social accueillant des adolescents, etc.

De ces modalités de recrutement exogames, il découle que les légitimités, dans leur ensemble, sont bouleversées. Les professionnels qui exercent dans ces structures (soin, travail social, etc.), se retrouvent dans l'obligation de reconsidérer ce qui, d'une certaine manière, allait de soi, dans la mesure où une partie du cadre n'étant pas mis en branle de manière aussi radicale qu'il ne l'est avec l'arrivée de ces personnes, radicalement étrangères au champ d'exercice. Ces personnes ne sont pas, en effet, organisées psychiquement à partir des organisateurs psychiques inconscients (Anzieu, 1980 ; Kaës, 1976, 1993), qui spécifient ces champs et ces secteurs d'activités. Elles peuvent, dès lors, imposer de nouvelles règles, énoncées comme obligatoires, sous le couvert mensonger d'un pseudo réel des contraintes budgétaires. 
Précisons qu'un tel cadrage pourrait laisser entendre que les filiations antérieures, dans ces établissements, ne posaient pas de problèmes majeurs, ce qui, bien entendu, serait un pur non-sens. L'un de nous a montré, au cours de ses travaux de recherche, que tout mouvement généalogique est facteur de mise en crise, et que, lors de tout passage généalogique, l'on a à faire à une déliaison majeure qui donne prise aux agir meurtriers (Gaillard, 2002). Ce qui est nouveau et spécifique à la période actuelle, c'est que les mouvements de recrutement de personnes, qui n'ont pas construit leurs étayages professionnels à partir de ce champ de la mésinscription et qui sont, donc, en position résolument exogames à l'égard des équipes dont elles prennent la direction, portent directement atteinte aux identités de ces mêmes institutions, potentialisant leurs fragilités intrinsèques (du fait même de leur tâche primaire, qui consiste à prendre en compte les fragilités identitaires des sujets qu'elles accueillent, accompagnent, etc., et des identifications que supposent ces liens). Les mouvements inhérents aux périodes de changement de responsables, la prédation et le rapt de la légitimité ne trouvent plus à être atténuées par des effets de culture, et l'on a affaire à une optimisation de la casse. La souffrance au travail et les agirs meurtriers de la professionnalité sont donc bien en passe de se généraliser.

\section{Le nouvel arrivant suspect de prédation}

Freud nous a familiarisé avec l'idée de l'indispensable renoncement pulsionnel, comme socle du lien social ${ }^{14}$. Une certaine abstinence est donc de mise, qui permet la construction de liens d'alliances (hors d'un pacte pervers), soit de faire équipe au sein des institutions. Les mutations actuelles donnent à croire à une subversion de ce socle du vivre ensemble. S'il n'existe pas de configuration, où ce renoncement peut être tenu pour acquis de façon définitive, il est, toutefois, la condition de l'exercice professionnel lui-même. Pour être à même de prendre des risques, dans le lien aux

14. Il s'agit là de l'une des « leçons » qui découlent de son élaboration mythique Totem et tabou (1912/1913), et des développements qu'il proposera dans Psychologie des foules et analyse du Moi (1921) différents « usagers » de ces institutions de la mésinscription, le professionnel doit pouvoir s'appuyer sur un lien de confiance avec son groupe d'appartenance ; il lui faut l'assurance de la non prédation, de la non rétorsion, soit, donc, un renoncement partagé. Le groupe est alors le garant de la limite et de la créativité de ses membres (individuellement et collectivement).

La cohésion des équipes de professionnels est, en effet, liée au sentiment que chacun contribue au narcissisme du groupe, au travers de son engagement auprès des « usagers »; simultanément, le groupe assure le narcissisme de chacun, en préservant sa professionnalité. « $\mathrm{Si}$ j'accepte de me perdre momentanément (en m'engageant avec tel résident, tel patient), je suis assuré que le groupe saura me tirer hors de la fascination, etc., saura m'aider à me redifférencier, à transformer les affects, etc. ». Or, au vu de la massivité des souffrances et des problématiques portées par les « usagers ", les professionnels échouent plus souvent qu'à leur tour. Dans les moments où le narcissisme n'est plus suffisamment assuré, la suspicion s'instaure. L'autre devient suspect de ne pas faire un effort à la mesure du mien, de ne pas souscrire au contrat de renoncement pulsionnel. La dérive est fréquente où un professionnel se met à penser que l'autre tire profit, de façon privilégiée, de la situation, au titre de sa propre économie psychique ; qu'il prélève sa prime de jouissance sur mon dos. Les figures et les étiquettes de " pervers » sont alors monnaie courante.

Au niveau des professionnels, tout nouvel arrivant est, d'emblée, suspect d'être en position de prédateur potentiel. Selon la place qu'il va être amené à occuper dans le groupe, il est suspect de capter, peu ou prou, la jouissance du groupe (voire de jouir du groupe lorsqu'il s'agit des changements de personnel qui touchent à des fonctions de cadre), propulsant les professionnels dans une imaginaire passivation, dès lors inacceptable. La tâche du groupe consiste à configurer le fond d'omnipotence et la violence prédatrice potentielle du nouvel arrivant, en les liant, dans l'urgence, à la psyché groupale déjà constituée. Le nouvel arrivant est mis dans une « urgence identificatoire » (Missenard, 1972), une urgence d'assignation.

Toute mise en place d'un " contrat narcissique » (Aulagnier, 1975) suppose une violence instituante. Dans les institutions de la mésinscription, le nouvel arrivant est mis en position d'incompétence, en position de « ne pas savoir y faire seul », et de devoir requérir l'aide et l'expérience de ceux qui sont déjà là. Il est mis dans l'obligation d'en passer par le groupe.

Lors de l'arrivée d'une personne venant occuper un poste " clef ", et lors des temps de passages généalogiques, ces craintes sont optimales, nous l'avons souligné. Celui qui est en position de responsabilité a fonction de représentance. Or, c'est la qualité du travail de chacun des professionnels qui contribue à la constitution d'un narcissisme et d'une créativité groupale. Le responsable peut capter cette prime narcissique, la canalisant de façon préférentielle au service de sa propre assise narcissique et de la vitesse de sa promotion. La qualité et la reconnaissance du travail fait par les professionnels peuvent s'en trouver fantasmatique-ment dérobées, dès que le responsable parvient à échapper à ces contrats et pactes antérieurs, et à s'imposer comme le détenteur de la nouvelle et, dès lors, unique légitimité. 


$$
\text { "Quand dire, c'est faire " " }
$$

L'occupation de postes de pouvoir potentialise une dérégulation narcissique, pousse à l'inflation, qu'il s'agisse du pouvoir hiérarchique ou du pouvoir de l'énoncé : ce pouvoir du « savoir » qui concerne directement les «psychistes » dans les établissements. Est ici désignée comme poste de pouvoir, toute place, sur la scène sociale, qui met le sujet en position de «visibilité », dans le même temps où elle accroit la fonction performative de sa parole : " quand dire, c'est faire » (Austin, 1962). Il s'agit de ces positions, où la parole de l'un peut valider ou invalider la position de l'autre. En ce sens, le pouvoir a trait à l'accroissement de la zone d'influence d'un sujet, et à son influence directe sur la place de l'autre. Si le pouvoir de nomination en constitue le paradigme, le pouvoir de l'interprétation n'est pas loin derrière.

Or, le pouvoir, nous l'avons souligné, est une puissante source de jouissance (narcissique, phallique et mortifère). Un vertige peut, dès lors, s'emparer du sujet, lorsque l'écart entre " dire et faire » se réduit drastiquement. Le sujet peut, ainsi, être saisi par l'éprouvé d'un illusoire effacement de la temporalité, de la limite et de la dépense (corporelle) qui lui est consubstantielle. « Dire », pour un dirigeant, fait acte, via le corps et la psyché d'un autre sujet (la « force de travail » du subordonné est ainsi orientée, en lien avec la tâche qu'il lui est demandé de réaliser). Le corps du sujet, en position de responsabilité, s'efface au profit de sa parole, sans qu'il n'y ait nécessité d'un déploiement de la temporalité, du passage par un processus qui en diffère la réalisation, à partir d'une mise en acte et de l'effort corrélé. L'engagement du sujet et le nécessaire passage par la limite du corps s'effritent. L'omnipotence refait alors surface ; «Sa majesté le bébé » (Freud, 1914) retrouvé peut,

15. Cette formule fait référence au titre de l'ouvrage fondateur de J. L. Austin (1962), dans sa traduction française de 1970, alors énoncer : « Je veux ! J'exige ! J'ordonne ! " L'interdit de prédation et le renoncement pulsionnel ne sont plus garantis.

\section{Disqualifications paradigmatiques}

Afin de donner une plus grande lisibilité à ces dynamiques, voici quelques propos d'une directrice administrative, issue du monde de l'industrie, quelques mois après sa prise de fonction dans une structure regroupant différents établissements (internats, Service d'éducation spécialisée et de soins à domicile, etc.) s'occupant d'adolescents présentant des « troubles de la personnalité »(selon les termes de l'agrément). $\mathrm{Au}$ cours d'une même réunion, elle fera usage de propos disqualifiant avec une aisance remarquable.

À l'une des trois psychologues, celle-ci étant originaire d'un pays de l'Est, et travaillant dans l'établissement depuis plusieurs années, elle dira : «Vous n'allez pas participer à la réunion avec les écoles. Lorsque vous parlez, on ne vous comprend pas ! »Au chef de service éducatif, à qui elle venait de proposer une responsabilité plus importante : "Vous n'allez plus participer aux réunions de supervision du centre $»$ (du lieu de son appartenance historique, cette personne ayant fait partie des membres fondateurs du projet), «Cela vous permettra de vous dégager affectivement !» $\mathrm{Au}$ médecin psychiatre, coordonnateur médical, anciennement responsable de l'un des établissements : « Dorénavant, c'est moi qui effectuerai les recrutements ; d'ailleurs, lors du dernier recrutement, vous avez fait une erreur, vous auriez dû voir que l'éducateur que vous avez retenu n'allait pas rester. Moi, je l'aurais vu dans son $\mathrm{CV} »$. À ceci, il convient d'ajouter que, pour la première fois de sa carrière, ce médecin s'est retrouvé contrôlé de manière infantilisante, la directrice ayant demandé, à la secrétaire, le relevé détaillé de l'ensemble des prises en charge du médecin psychiatre, sur une période de plusieurs mois. La secrétaire, se vivant comme otage de ce rapport de force, ne trouvera pas d'autre issue que de se retrouver en arrêt maladie.

Nous pourrions multiplier les citations à l'envi, tant nous rencontrons, dans ces structures, des responsables désinhibés dans l'exercice de leur pouvoir. Les propos y sont caricaturaux dans la violence de la disqualification à l'œuvre. Procédant à un véritable rapt de la légitimité, le responsable met chacun des acteurs en faute, eu égard aux nouvelles normes dont il détermine les critères. La déliaison mortifère se donne, dès lors, libre cours, entraînant parfois de véritables hémorragies de personnel, à moins que ceux-ci ne parviennent à circonscrire la destructivité dont ils sont le lieu, dans la mise en place de contre-pouvoir efficient. L'énergie requise pour la construction de ces rapports de force, se joue, toutefois, au détriment de la tâche primaire ; les « usagers » (patients, résidents, etc.) pouvant, bien entendu, se retrouver en position d'otages de ces tensions. 


\section{LA CONFLICTUALITÉ CONTRE \\ L'UNIFORME}

La menace de « l'uniforme »- la menace d'une standardisation, sous le primat de l'utilitarisme et de « l'idéologie de la transparence » (Pinel, 2008) -, fait surgir le spectre de la stérilisation de la créativité des professionnels, cette disposition indispensable aux prises en charge de soin et d'accompagnement. La tentation actuelle, qui s'offre aux professionnels, est, dès lors, celle d'une «pensée prête à penser » déshumanisante. Peut-être ne restera-t-il (sous peu) d'autre alternative que d'emprunter les voies de la désobéissance, pour maintenir ouverte la possibilité d'une pensée vivante et d'un acte « habité ».

Dans un tel contexte, il importe de se rendre attentif à ce qui peut être préservé (voire fabriqué) comme dynamiques de conflictualité ${ }^{16}$. C'est, en effet, la pulsion anarchiste (Zaltzman, 1998), l'aspect de la déliaison qui est au service de la vie, qui permet de restaurer de la différence et de lutter contre la transformation de l'institution en establishment ${ }^{17}$ (Bion, 1959), et de la groupalité institutionnelle en appartenance sectaire (Diet, 2008). La différence entre les professionnels doit pouvoir se mettre en scène et se déployer dans le même temps où se développent des processus d'appartenance et de pensée autoréflexifs (Gaillard et coll., 2009), sources de créativité.

Lorsque la conflictualité sous-tend les appartenances, elle autorise les professionnels à se vivre comme reliés, et à se différencier. La mise en place d'un lien groupal, respectueux de chacun, préserve

16. En l'absence de conflictualité externe au sein d'une société, la conflictualité, condition du fonctionnement de l'appareil psychique, ne peut se maintenir, selon Guy Laval (2002).

17. L'apport de W. Bion (1961) et sa suite de nombre de chercheurs qui se sont penchés sur la dynamique institutionnelle (Enriquez, 1987 ; Kaës, 1987 ; Fustier, 1987 ; Rouchy, 1998 ; etc.), nous a permis de penser le processus de rigidification, qui transforme l'établissement en une structure centrée sur sa pérennisation et fonctionnant sous le primat de «l'organisation».

le groupe d'un éclatement où la conflictualité bascule en conflit. Tout groupe professionnel a besoin de s'éprouver comme suffisamment fécond. Lorsque le travail, auprès des différents « usagers ", donne à vivre, à chacun, un sentiment de créativité, le narcissisme s'y nourrit de façon suffisante. Les professionnels ont alors moins besoin de "se payer sur la bête ", manière de dire qu'ils sont moins tentés de jouer leur propre destructivité dans le lien à l'usager et dans le lien aux différents collègues.

\section{Reconnaître la présence de Thanatos}

Nous avons trop rapidement souligné comment l'idéologie managériale sous-tend les mutations sociales actuelles (mises en concurrence de l'ensemble des acteurs et des établissements, défis de l'excellence, destruction des acquis sociaux et des alliances groupales qu'elles supposaient, etc.), et met en œuvre une véritable déconstruction des légitimités et des modalités de liens antérieurs. Dans un tel contexte, il convient de se rappeler que le travail d'appropriation subjective, la construction du sujet, s'inscrit dans du temps long, et que les identifications prennent ancrage dans l'histoire, tout comme les symptômes. Ce « management gestionnaire » participe à la méconnaissance du primat de la pulsion de mort.

L'un des enjeux actuels réside dans la capacité des groupes institués à se configurer dans un appareillage groupal, qui reconnaisse sa place à ce « travail de la mort ». Les institutions de la mésinscription sont des laboratoires d'expérimentation. Elles sont aux premières lignes dans le travail de maillage, dans la restauration des liens souffrants. C'est toujours dans les marges que la créativité trouve son terreau le plus fécond. Le symptôme est la part du sujet qui refuse de se laisser réduire (dans l'emprise ou l'abandon). Or ce sont, précisément, de multiples symptômes qui s'actualisent sur la scène sociale dont s'occupent les institutions de la mésinscription. Gageons que, dans la crise généalogique actuelle, le sujet continuera à revendiquer sa part de créativité et saura faire pièce à la menace de standardisation et de stérilisation en cours. Gageons que la groupalité obligée des professionnels, aux prises avec le mortifère du symptôme, jouera comme contrainte, donnant forme à de nouvelles assises narcissiques, reconnaissant la présence, au centre, de Thanatos. 


\section{RÉFÉRENCES}

ANZIEU (Didier).- Le groupe et l'inconscient, Paris, Dunod, 1980.

ARENDT (Hanna).- Les origines du totalitarisme, Eichmann à Jérusalem [1951], Paris, Gallimard, 1956, 2002.

ASSOUN (Paul-Laurent).- La psychanalyse à l'épreuve du pouvoir, Analyse et réflexion sur le pouvoir, II, Paris, Ellipses, 1994, p. 59-71.

AULAGNIER (Piera).- La violence de l'interprétation. Du pictogramme à l'énoncé, Paris, Presses universitaires de France, 1975.

AUSTIN (John Langshaw).- How to do things with words [1962], trad. fr., Quand dire, c'est faire, Paris, Seuil, 1970.

BARTHELEMY (Sophie), GIMENEZ (Guy), PEDINIELLI (Jean-Louis).- De l'enveloppe soignante à l'enveloppe sociale, Revue française de psychologie et de psychiatrie médicale, 108, 2007, p. 6-11.

BENASAYAG (Miguel).- Le mythe de l'individu, Paris, La découverte, 1998.

BION (Wilfried Ruprecht).- Attaque contre la liaison [1959], dans Bion (W. R.), Réflexion faite, Paris, Presses universitaires de France, 2001, p. 105-123.

BION (Wilfried Ruprecht).- Experiences in groups [1961], trad. fr., Recherches sur les petits groupes, Paris, Presses universitaires de France, 1987.

BLEGER (José).- Psychanalyse du cadre psychanalytique [1966], dans Kaës (R.), Missenard (A.), Kaspi (D.), Guillaumin (J.), Bleger (J.), Jaques (E.), Crise, rupture et dépassement, Paris, Dunod, 1979, p. 255-274.

CASTORIADIS (Cornelius).- La montée des insignifiances, les carrefours du labyrinthe 4, Paris, Seuil, 1996.

DIET (Emmanuel).- L'homme procédural. De la perversion sociale à la désubjectivation aliénante, Connexion, 79, 1, 2003, p. 11-28.

DIET (Emmanuel).- La groupalité sectaire : emprise et manipulation, dans Lecourt (E.), Modernité du groupe dans la clinique psychanalytique, Toulouse, Érès, 2008,

p. 149-164. DOUVILLE (Olivier).- L'“identité/altérité", fractures et montages. Essai d'anthropologie clinique, dans

Kaës (R.), Différence culturelle et souffrances de l'identité, Paris, Dunod, 1998, p. 21-44. DUFOUR

(Dany-Robert).- La condition subjective dans les sociétés démocratiques, dans Assoun (P.-L.), Zafiropoulos (M.),

L'anthropologie psychanalytique, Paris, Anthropos, 2002, p. 25-47. ELIAS (Norbert).- La société des individus,

Paris, Fayard, 1991. ENRIQUEZ (Eugène).- Le travail de la mort dans les institutions, dans Kaës (R.), L'institution

et les institutions, Paris, Dunod, 1987, p. 62-94. ENRIQUEZ (Eugène).- Le groupe, lieu d'oscillation

entre repli identitaire et travail de l'interrogation, Revue française de psychanalyse, 63, 4, 1999, p. 801-814.

FREUD (Sigmund).- Totem et tabou [1912/1913], Paris, Gallimard, 1993.

FREUD (Sigmund).- Pour introduire le narcissisme [1914], dans Freud (S.), La vie sexuelle, Paris, Presses universitaires de France, 1969, p. 81-105.

FREUD (Sigmund).- Au-delà du principe de plaisir [1920], dans Freud (S.), Essais de psychanalyse, Paris, Payot, 1981, p. 41-115.

FREUD (Sigmund).- Psychologie des foules et analyse du Moi [1921], dans Freud (S.), Essais de psychanalyse, Paris, Payot, 1981, p. 119-217.

FREUD (Sigmund).- XXXI leçon. La décomposition de la personnalité psychique [1932], dans Freud (S.), Nouvelle suite des leçons d'introduction à la psychanalyse, Euvres complètes XIX, Paris, Presses universitaires de France, 1995, p. 140-163.

FREUD (Sigmund).- Pourquoi la guerre ? [1933], dans Freud (S.), Résultats, idées, problèmes II, Paris, Presses universitaires de France, 1985, p. 203-215.

FURTOS (Jean).- De la précarité à l'auto-exclusion, Paris, Éditions de la rue d’Ulm, 2009.

FUSTIER (Paul).- L'infrastructure imaginaire des institutions. À propos de l'enfance inadaptée, dans Kaës (R.), Bleger (J.), Enriquez (E.), Fornari (F.), Fustier (P.), Roussillon (R.), Vidal (J.-P.), L'institution et les institutions, Paris, Dunod, 1987, p. 131-156.

FUSTIER (Paul).- Le travail d'équipe en institution. Clinique de l'institution médico-sociale et psychiatrique, Paris Dunod, 1999.

GAILLARD (Georges).- La généalogie institutionnelle et les écueils du travail d'historisation entre filicide et parricide, Connexions, 76, 2002, p. 125-141.

GAILLARD (Georges).- Se prêter à la déliaison. Narcissisme groupal et tolérance au féminin dans les institutions, Connexions, 90, 2008, p. 11-25.

GAILLARD (Georges).- Confidentialité et transparence, confiance et suspicion : la précarité des liens dans les groupes institués, dans Katz (M.) et coll. Confidentialité et clinique psychanalytique, Paris, In Press (à paraître).

GAILLARD (Georges), PINEL (Jean-Pierre).- L'analyse de la pratique en institution : un soutien à la professionnalité dans un contexte d'emprise du modèle gestionnaire ?, Nouvelle revue de psychosociologie, 11, 2, 2011,

p. 85-103. GAILLARD (Georges), PINEL (Jean-Pierre), DIET (Emmanuel).- Autoréflexivité et conflictualité dans les groupes institués, Nouvelle revue de psychosociologie, 8, 2009, p. 199-213. GAUCHET (Marcel).- La religion dans la démocratie, parcours de la laïcité, Paris, Gallimard, 1998. GIRARD (René).- Le bouc émissaire, Paris, 
Grasset, 1982.

GREEN (André).- Narcissisme de vie, narcissisme de mort, Paris, Les éditions de minuit, 1983.

HASSOUN (Jacques).- Les contrebandiers de la mémoire, Paris, Syros, 1994.

HENRI (Alain-Noël).- Le secret de famille et l'enfant improbable, dans Mercader (P.), Henri (A.-N.), La formation en psychologie filiation bâtarde, transmission troublée, Lyon, Presses universitaires de Lyon, 2004,

p. 193-303.

HENRI (Alain-Noël).- Penser à partir de la pratique. Rencontre avec Alain-Noël Henri, Toulouse, Érès, 2009.

KAËS (René).-L'institution et les institutions, Paris, Dunod, 1987.

KAËS (René).- L'appareil psychique groupal [1976], Paris, Dunod, 2010.

KAËS (René).- Le groupe et le sujet du groupe, Paris, Dunod, 1993.

KAËS (René).- Souffrance et psychopathologie des liens institués, une introduction, dans Kaës (R.), Pinel

(J.-P.),Kernberg (O.), Correale (A.), Diet (E.), Duez (B.), Souffrance et psychopathologie des liens institutionnels, Paris, Dunod, 1996, p. 1-47.

KAËS (René).- Tyrannie de l'idéal et de l'idole. La position idéologique, dans Ciccone (A.) et coll., Psychanalyse du lien tyrannique, Paris, Dunod, 2003, p. 69-104.

KANT (Emmanuel).- Sur le mal radical dans la nature humaine [1792], Paris, Éditions de la rue d'Ulm, 2011.

LASCH (Christopher).- La culture du narcissisme [1979], Castelnau-le-Lez, Éditions Climats, 2000. LAVAL

(Guy).-Bourreaux ordinaires. Psychanalyse

du meurtre totalitaire, Paris, Presses universitaires de France, 2002.

LEGENDRE (Pierre).- La fabrique de l'homme occidental, Paris, Mille et une nuits et Arte éditions, 1996.

LÉVIN

$\mathrm{S}$ (Emmanuel).-Éthique et infini, Paris, Arthème Fayard, 1982.

LYOTARD (Jean-François).- La condition de l'homme moderne, Paris, Les éditions de minuit, 1979.

MELMAN (Charles).- L'homme sans gravité. Jouir à tout prix, Paris, Denoël, 2002.

MISSENARD (André).- Identification et processus groupal, dans Missenard (A.), Le travail psychanalytique dans les groupes, I, Paris, Dunod, 1972, p. 217-250.

PINEL (Jean-Pierre)-- Emprise et pouvoir de la transparence dans les institutions spécialisées, Revue de psychothérapie psychanalytique de groupe, 51. Pouvoir et emprise dans les groupes, 2008, 2, p. 33-48.

PONTALIS (Jean Bertrand).-En marge des nuits, Paris, Gallimard, 2010.

ROUCHY (Jean-Claude).- Le groupe espace analytique. Clinique et théorie, Toulouse, Érès, 1998.

VOLTAIRE.- Zadig ou la destinée, Londres (Amsterdam), Pour la compagnie, 1748.

ZALTZMAN (Nathalie).- De la guérison psychanalytique, Paris, Presses universitaires de France, 1998.

ZALTZMAN (Nathalie).- De surcroît... ? Le travail de culture ? La guérison ? L'analyse elle-même ?, dans Green (A.), Le travail psychanalytique, Paris, Presses universitaires de France, 2003, p. 211-219 et p. 233-239 